\title{
Detection Algorithm of Porosity Defect on Surface of Micro-precision Glass Encapsulated Electrical Connectors
}

\author{
Qunpo Liu ${ }^{1, *}$, Manli Wang ${ }^{1}$, Gaowei Wang ${ }^{1}$, Ruxin Gao ${ }^{1}$, Naohiko Hanajima ${ }^{2}$ \\ ${ }^{1}$ Department of Robotics Engineering, Henan Polytechnic University, 2001 Century Avenue, Jiaozuo, Henan 454003, P. R. China \\ ${ }^{2}$ College of Information and Systems, Muroran Institute of Technology, 27-1 Mizumoto-cho, Hokkaido, Muroran-shi, Hokkaido 050-8585, Japan
}

\author{
ARTICLE INFO \\ Article History \\ Received 15 October 2019 \\ Accepted 13 June 2020 \\ Keywords \\ Micro-precision glass encapsulated \\ electrical connectors \\ manual inspection \\ surface defect inspection \\ feature extraction
}

\begin{abstract}
A miniature precision glass encapsulated electrical connectors introduced by glass powder and metal wires through a special complicated process. Aiming at the porosity defects on the surface, a defect detection algorithm propose based on threshold segmentation and feature extraction. Pre-operation, global threshold segmentation processing and feature extraction (based on area, circularity aspect ratio, compactness, and contour length) are preformed to detect the defects. Experimental results show that the algorithm can accurately identify porosities defects.
\end{abstract}

(C) 2020 The Authors. Published by Atlantis Press B.V. This is an open access article distributed under the CC BY-NC 4.0 license (http://creativecommons.org/licenses/by-nc/4.0/).

\section{INTRODUCTION}

The micro-precision glass encapsulated electrical connectors is mainly used to connect a module, fix its wires and insulate it from the "tube shell", as shown in Figure 1. The glass encapsulated electrical connector structure is composed of three parts: the connector, the high-temperature glass layer, and the low-temperature glass layer in order from the inside. Due to the low density of the low-temperature glass layer on the outside of the electrical connector, which is fluffy, and the material contains lead, the operating temperature during the sealing process is abnormal (such as too high or too low), and the accuracy of the equipment is not high or malfunctions. As a result, part of the surface structure of the low-temperature glass layer is not compact enough, thereby causing porosity and crack defects. Therefore, the detection of porosity and crack defects on the surface of glass encapsulated electrical connectors is mainly the detection of defects at the low temperature glass welded to the outside. This article is mainly concerned with porosity defect.

The glass encapsulated electrical connector has a small volume (about $2.85 \mathrm{~mm}$ in diameter) and requires high accuracy $(<0.01$ $\mathrm{mm})$. At present, the detection of defects such as porosities and cracks on the surface of the glass encapsulated electrical connector achieve manually by combining with a magnifying glass. However, people who work for a long time are prone to eye fatigue and labor intensity [1]. There are too many subjective factors during manual inspection. Over-fatigue caused by high-intensity work and fast inspection speed may lead to missed inspection or false inspection. As a result, the defective encapsulated electrical

"Corresponding author. Email: lqpny@hpu.edu.cn connector assembly applied, resulting in poor or even unusable product quality and need to rework, causing huge economic losses. In addition, glass encapsulated electrical connectors are in great demand in some respects and show expansive growth. However, the automatic detection of porosity defects on the surface of connectors at home and abroad is still blank. Therefore, the automatic detection of porosities defects on the surface of glass encapsulated electrical connectors has become a major problem that needs to solve urgently.

For the research of surface defect detection, the Wang et al. [2], Mei et al. [3] and so on use deep learning or support vector machine to detect the surface defect of the object, but the calculation time is long and the sample demand is large. The Chen et al. [4], Wang et al. [5] conducts defect detection by extracting features such as geometric features of defect areas on the surface of objects, and then identifies and classifies the defects. The method is simple, fast and practical. Based on the characteristics of porosity defects on the surface of glass encapsulated electrical connectors, a defect detection of surface algorithm for micro-precision glass insulated encapsulated electrical connectors based on threshold segmentation and feature extraction was proposed.

\section{DETECTION ALGORITHM BASIC STRUCTURE}

The algorithm uses Halcon HDevelop (18.11) to perform preprocessing operations such as background removal, and $G$ channel acquisition of the image with defects. Then, these two defects are detected using global threshold segmentation processing and feature extraction methods based on area, circularity, aspect ratio, 

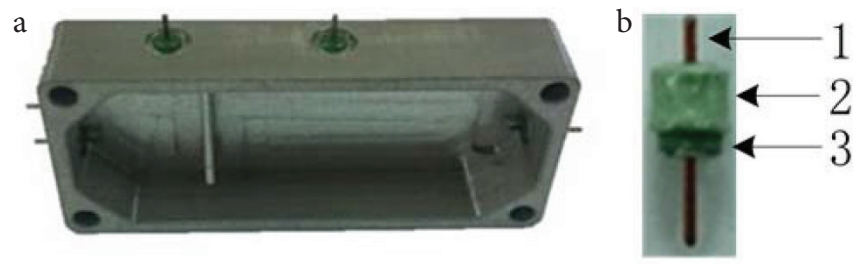

Figure 1 Glass terminal and its installation. (a) Glass terminal installation. (b) The micro-precision glass encapsulated electrical connectors.

compactness, and contour length, and the characteristic parameters of the defect area are calculated and analyzed. Finally, the classification is based on the difference between the porosity and crack defect characteristics, and the specific process is image preprocessing, defect detection, feature parameter analysis, and final classification decision.

\section{IMAGE PRE-PROCESSING}

\subsection{Background Removal}

The pictures acquire with an image-measuring instrument and the colored image is $640 \times 480$.

In order to facilitate defect detection, it is necessary to separate the end surface area of the glass encapsulated electrical connector from the background to reduce the interference. Since blue does not occur naturally in glass encapsulated electrical connectors and is one of the three channels in the RGB color space, it is easier to segment the foreground area from a blue background than other colors [6]. In order to verify the feasibility of this method from the aspect of practical application, and use a large number of samples with different colors for experimental verification and theoretical analysis. By manually changing the background to ten common colors such as black, gray, white, red, orange, yellow, green, cyan, blue, and purple, the experimental verification and comparative analysis of glass encapsulated electrical connector defect samples were performed. In order to meet the requirements of practical applications, dope the background color appropriately, and it finally find that when the background is biased to pure blue, it can completely meet the requirements of removing the main background. In addition, because the color of the upper-left character of the collected picture is green, which is different from the main background blue color. It is necessary to fill the character area (that is, the area from the image origin to one-sixth long and one-third wide) to the purpose of process of background which include color image to Hexcone Model (HSV) space, blue threshold mask and bitwise negation to generate the target area mask, make the original image and itself bitwise operate in the target area mask, character area color fill. The removal result is shown in Figure 2. Where, the range of values of $\mathrm{H}, \mathrm{S}$, and $\mathrm{V}$ when generating mask in Figure 2 is: H: [100, 124], S: [43, 255], V: [46, 255].

\subsection{Obtaining the Glass Encapsulated Electrical Connector G Channel}

During the implementation of detection technology of machine vision, due to the requirements of detection speed and algorithm
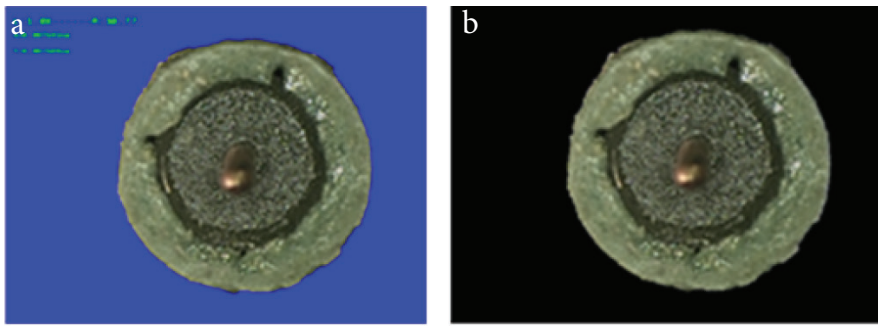

Figure 2 The removal result of background. (a) Stomatal defect sample. (b) Background removal of the sample.

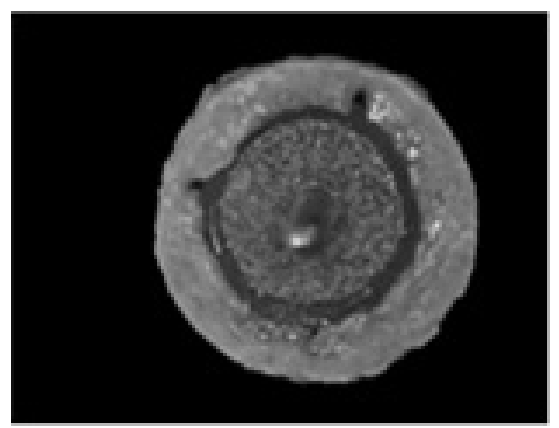

Figure 3 G channel for extracting defective samples.

implementation, it is often necessary to convert color images into grayscale images for processing. For the convenience of detection, the three-channel color image is converted into six single-channel grayscale images of $\mathrm{R}, \mathrm{G}, \mathrm{B}, \mathrm{H}, \mathrm{S}$, and I. After testing and observation, it is found that the defect part of the glass channel of the $G$ channel is more obvious, so the image of the $G$ channel is selected as the detection map, and the $\mathrm{G}$ channel of the glass encapsulated electrical connector is shown in Figure 3.

\section{DEFECT DETECTION}

The porosity defect on the surface of the glass encapsulated electrical connector are more clearly identified by color, and gray value is lower than other surrounding areas, and they are approximately black. Therefore, global threshold segmentation and feature-based detection and recognition methods use for the detection of porosity defects.

\subsection{Threshold Segmentation}

Global threshold segmentation perform on the pre-processed glass encapsulated electrical connector image. The threshold segmentation is shown in Eq. (1).

$$
G_{\min } \leq g(x, y) \leq G_{\max }
$$

where, $g(x, y)$ is the gray-value of the image, $G_{\min }$ and $G_{\max }$ is the minimum pixel value (15 here) and the maximum pixel value ( 35 here). That is, the area formed by the gray-value of the image between the minimum pixel value and select the maximum pixel value in the image as the defect area to select.

After the above color feature processing, the detection range of glass encapsulated electrical connector defects can reduce, and 
the distribution of suspicious defects can be roughly determined. Then, the defect area can be detected by the geometric features of the defects. The segmentation result is shown in Figure 4 .

\subsection{Detection and Identification of Defect Geometric Features}

According to the distribution of suspicious defects of the glass encapsulated electrical connector, finally determining the porosity defect also requires multiple features for detection and identification. Common defect features in defect detection are area, circularity, perimeter, length, width, ratio of major axis to minor axis, defect center of gravity, circular or stripe defects, average grayscale and contrast, etc. This article mainly uses area, circularity, aspect ratio and compactness for feature selection.

\subsubsection{Defect area}

The area of a defect can be simply defined as the number of pixels contained in the boundary of the defect, which is only related to the size of the target area, and has nothing to do with the gray-value of the pixels at each point. The area is determined as shown in Eq. (2).

$$
A_{s}=\sum_{(x, y) \in s} f(x, y)
$$

where, $s$ is the target area, $f(x, y)$ is pixel value, $A_{s}$ is object area.

\subsubsection{Roundness of defects}

It can be defined as the ratio of the area to the maximum area, as shown in Eq. (3).

$$
C=\frac{A_{s}}{\pi d_{\max }^{2}}
$$

where, $d_{\max }$ is the maximum distance from the defect center to the area boundary.

\subsubsection{Aspect ratio of defects}

The aspect ratio refers to the ratio of the major axis to the minor axis of the circumscribed ellipse of the region, as shown in Eq. (4).

$$
R_{t}=\frac{R_{a}}{R_{b}}
$$

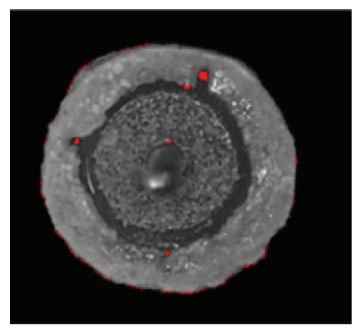

Figure 4 Threshold segmentation map (red area is a suspected defect area). where, $R_{a}$ and $R_{b}$ are the major and minor axes of the circumscribed ellipse, respectively.

\subsubsection{Tightness of defects}

Tightness is a form factor that represents the tightness of a region. The tightness of the circle is 1 , if the area is long, the shape factor is greater than 1, and the longer the shape factor, the larger the shape factor, as shown in Eq. (5).

$$
C_{t}=\frac{L^{2}}{4 \pi A_{s}}
$$

where, $L$ is the outline length of the area.

\subsubsection{Defect contour length}

The contour length refers to the total length of the contour line at the boundary of the specified area, that is, the sum of the distances of the pixels on the edge of the contour. Among them, the distance between two adjacent contour points parallel to the coordinate axis is 1 , and the distance on the diagonal is $\sqrt{2}$.

\subsubsection{The characteristic values for identification}

The characteristic values of feature detection and identification of stomatal defect are shown in Table 1.

The glass encapsulated electrical connector defect detection results are shown in Figure 5.

\section{EXPERIMENT AND RESULTS}

In order to verify the feasibility of the algorithm, three samples with defects were randomly selected for experiments, as shown in Figure 6.

Table 1 Ranges of characteristic values of porosity defects

\begin{tabular}{lc}
\hline Defect type & Porosity \\
\hline$A_{s}\left(\mathrm{~mm}^{2}\right)$ & {$[0.0015,0.05]$} \\
$C$ & {$[0.25,1]$} \\
$R_{t}$ & {$[1.0,1.6]$} \\
$C_{t}$ & {$[1.0,2.0]$} \\
Contour length $(\mathrm{mm})$ & {$[0.05,0.60]$} \\
\hline
\end{tabular}

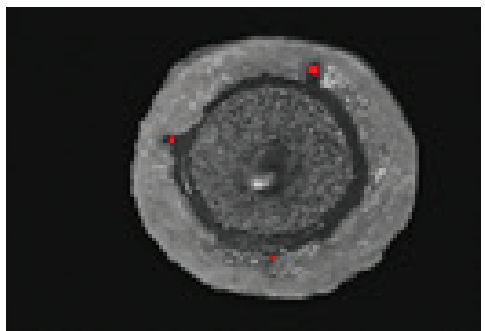

Figure 5 Defect detection results (red areas are defects). 

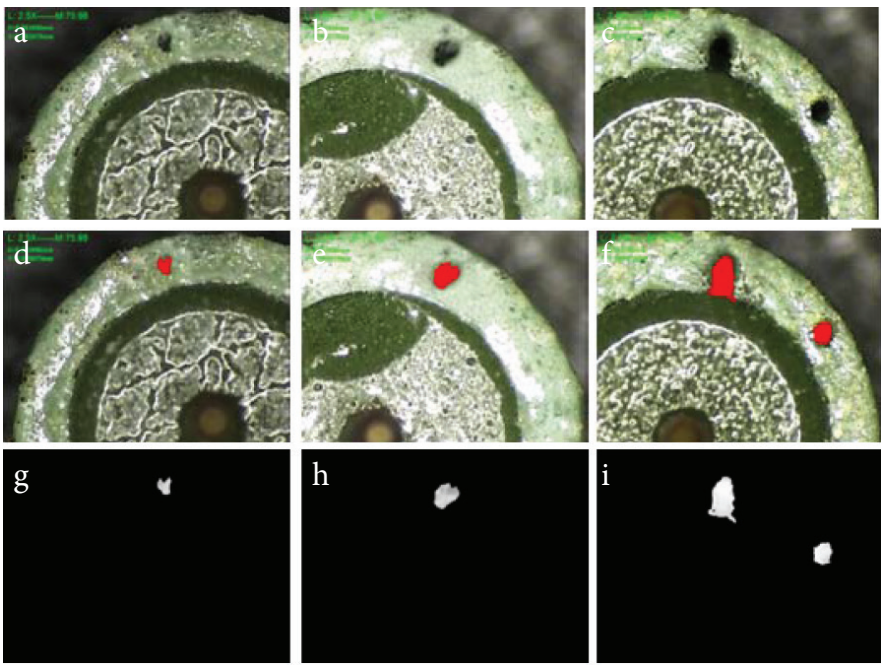

Figure 6 Defect detection results. (a) Sample-S1. (b) Sample-S2. (c) Sample-S3. (d) Defect area labeling in S1. (e) Defect area labeling in S2. (f) Defect area labeling in S3. (g) Defect-S1-p1. (h) DefectS2-p1. (i) Defect-S3-p1 and S3-p2.

Table 2 Characteristic values of porosity defects

\begin{tabular}{lcccc}
\hline Characteristic type & S1-p1 & S2-p1 & S3-p1 & S3-p2 \\
\hline$A_{s}\left(\mathrm{~mm}^{2}\right)$ & $7.7 * 10^{-3}$ & $21.1 * 10^{-3}$ & $38.2 * 10^{-3}$ & $15.4 * 10^{-3}$ \\
$C$ & 0.53 & 0.67 & 0.37 & 0.68 \\
$R_{t}$ & 1.21 & 1.13 & 1.25 & 1.05 \\
$C_{t}$ & 1.62 & 1.28 & 1.86 & 1.46 \\
Contour length $(\mathrm{mm})$ & 0.18 & 0.24 & 0.26 & 0.21 \\
\hline
\end{tabular}

The characteristic values of defects in experiment samples are shown in Table 2.

As shown in Table 2, it can be seen from the experimental results that the algorithm proposed in this paper can extract the porosity defect areas. And it can identify porosity defects based on characteristic parameters of area, roundness, aspect ratio, tightness, and contour length.

\section{CONCLUSION}

Based on the detection of porosities on the surface of glass encapsulated electrical connectors, a surface defect detection algorithm based on threshold segmentation and feature extraction is proposed based on the characteristics of the abnormal texture of the end surfaces. The background is removed by image pre-processing, and the defect can be obtained by threshold segmentation. Then, through the characteristics of area, roundness, aspect ratio, tightness, and contour length, the detection of porosity defects on the surface is realized. Laid the foundation for automatic quality inspection and assembly.

\section{CONFLICTS OF INTEREST}

The authors declare they have no conflicts of interest.

\section{ACKNOWLEDGMENTS}

This work is partially supported by National Key Research and Development Project (2016YFC0600906), Innovation Scientists and Technicians Troop Construction Projects of Henan Province (CXTD2016054) and also supported by Innovative Scientists and Technicians Team of Henan Provincial High Education (20IRTSTHN019).

\section{REFERENCES}

[1] Z.T. Shen, J.P. Chen, H.D. Du, R. Li, Research and application of insulator RTV coating quality inspection method based on machine vision, Insulat. Surge Arresters 285 (2018), 239-246 (in Chinese).

[2] T. Wang, Y. Chen, M. Qiao, H. Snoussi, A fast and robust convolutional neural network-based defect detection model in product quality control, Int. J. Adv. Manuf. Technol. 94 (2018), 3465-3471.

[3] S. Mei, H. Yang, Z. Yin, An unsupervised-learning-based approach for automated defect inspection on textured surfaces, IEEE Trans. Instrum. Measure. 67 (2018), 1266-1277.

[4] Y.Y. Chen, Y. Wang, H.X. Ye, H.T. Wu, Z.S. Cui, Research on the defect detection of machine vision toughened glass insulator bubble, Autom. Instrum. 31 (2010), 51-53+56 (in Chinese).

[5] S.H. Wang, Y.G. Cai, H. Cai, Research on glass surface defect detection algorithm, J. Dongguan Univ. Technol. 25 (2018), 45-49 (in Chinese).

[6] D. Zhang, D.J. Lee, B.J. Tippetts, K.D. Lillywhite, Date maturity and quality evaluation using color distribution analysis and back projection, J. Food Eng. 131 (2014), 161-169.

\section{AUTHORS INTRODUCTION}
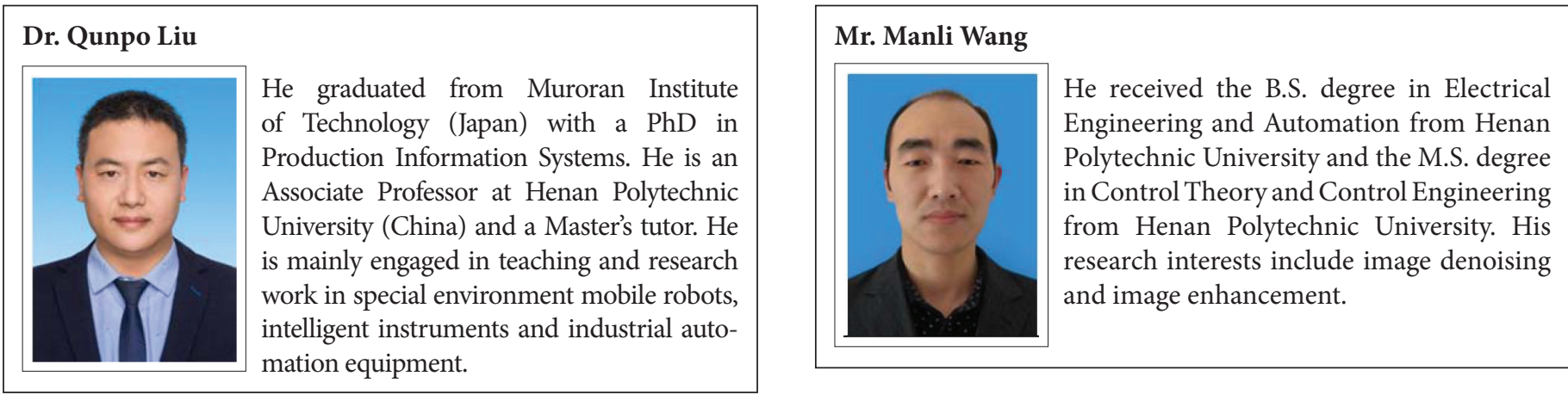


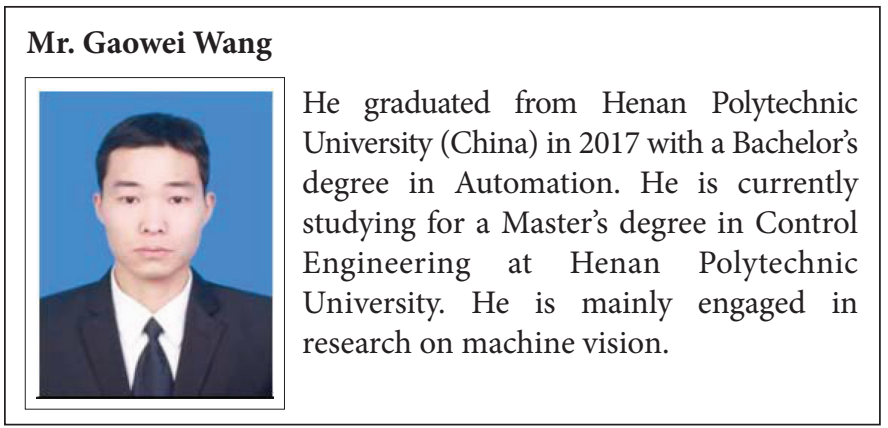

\section{Dr. Ruxin Gao}

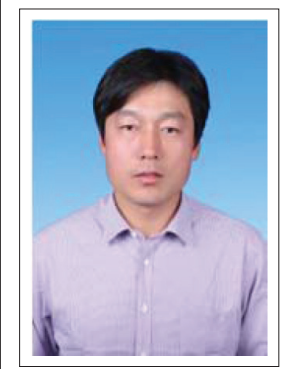

He graduated from Huazhong University of Science and Technology (China) with a $\mathrm{PhD}$ in Pattern Recognition and Artificial Intelligence. Now he is an Associate Professor at Henan Polytechnic University (China). He is mainly engaged in teaching and research work in image processing, machine vision and artificial intelligence.

\section{Prof. Naohiko Hanajima}

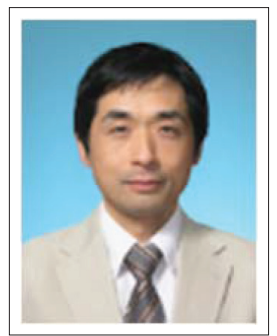

He graduated from the Hokkaido University (Japan) of Technology in Japan with a PhD. He is a Professor at the College of Information and Systems at Muroran Institute of Technology (Japan). He is mainly engaged in robotics and intelligent equipment. 